\title{
Recurrent, bilateral, and metastatic pheochromocytoma in a young patient with Beckwith-Wiedemann syndrome: A genetic link?
}

\author{
Tiffany Caza, MD, PhD'; Jared Manwaring, $M D^{2}$; Jonathan Riddell, $M D^{2}$
}

'Department of Pathology; '2Department of Urology; Upstate Medical University, Syracuse, NY, United States

Cite as: Can Urol Assoc J 2017; 11 (5):E240-3. htrtp://dx.doi.org/10.5489/cuaj.4297

Published online May 9, 2017

\section{Introduction}

Beckwith-Wiedemann syndrome (BWS) is a genetic disorder at chromosome $11 \mathrm{p} 15$ that leads to increased activity of insulin-like growth factor-2 (IGF2) and reduced activity, with no active copy of the inhibitor of cell proliferation, CDKN1C, resulting in excessive growth and increased risk of tumour formation. Isolated cases of patients with BeckwithWiedemann syndrome and pheochromocytoma are reported in the literature; however, none have described a molecular or cytogenetic evaluation for associated genetic abnormalities.

We present a case of an adolescent female with BeckwithWiedemann syndrome who developed recurrent, bilateral, and metastatic pheochromocytoma despite low-risk histopathology. Genotyping studies, which evaluated for genetic predisposition to pheochromocytoma or paraganglioma (PHEO/PGL), including the PGLNext sequencing panel of 12 associated genes, and a whole genome comparative genome hybridization microarray, were performed. Genomic studies were unexpectedly negative. Additionally, the histopathology of the PHEO/PGL of our patient had low-risk features, which is unusual in cases of metastases, occurring in less than $4 \%$ of cases. ${ }^{1}$

This case suggests that the BWS in itself could predispose to formation of a PHEO/PGL phenotype with aggressive behaviour. The following manuscript summarizes the case report, reviews pertinent literature, and proposes a possible mechanism for this association.

\section{Case report}

A 15-year-old female with BWS presented with palpitations, dizziness, near-syncope, and hypertension. She was found to have elevated plasma metanephrine levels (normetanephrine $2.42 \mathrm{nmol} / \mathrm{L}$ ). Computed tomography (CT) imaging revealed a large tumour in the left adrenal gland (Fig. 1). Review of systems was positive for headache, anxiety, sleep disturbance, urinary urgency, enuresis, back pain, and bilateral knee pain. Her past medical history included several complications of BWS, including a cystic pancreatic tumour (subtotal pancreatectomy in infancy), congenital hemihypertrophy with leg length discrepancy (osteotomy age 15), hyperinsulinemic hypoglycemia, and bilateral optic nerve edema. Family history was negative for genetic disorder or malignancy. Birth history was positive for preterm delivery (35 weeks) with neonatal hypoglycemia. She did not use tobacco, alcohol, or other substances. She was on no regular medication and had no drug allergies.

After blood pressure normalization with doxazosin, she underwent open adrenalectomy and retroperitoneal lymph node sampling. A subcentimeter suspicious nodule in the right adrenal was palpated and felt to be negative. The surgery was complicated by intraoperative bleeding and hypotension. Postoperatively, palpitations and dizziness resolved. The patient became normotensive and plasma metanephrines improved, but did not return to normal.

Pathology of the left adrenal gland revealed a 4 × 3.5 × 3.0 $\mathrm{cm}$ pheochromocytoma. The tumour showed vascular stroma with reactive changes and was without necrosis, cellular pleomorphism, or increased mitotic rate (Fig. 1). The tumour was encapsulated and completely excised. From the lymph node resection, a para-aortic tumour measured $2 \times 1.3 \times 0.9 \mathrm{~cm}$ with no lymph node parenchyma. S100 immunohistochemistry highlighted sustentacular cells, consistent with a paraganglioma. The presence of sustentacular cells is associated with benign behaviour; however, this finding has poor specificity and cannot reliably rule out metastasis. Synchronous disease was preferred, as the tumour showed well-differentiated histology and had no features of metastatic potential.

The grading system for adrenal pheochromocytoma and paraganglioma (GAPP) can be used to predict metastasis, ${ }^{2,3}$ and is helpful because metastatic disease is required for malignancy and $60 \%$ of metastases are asynchronous. Points are scored for the histological pattern, tumour cellularity, comedonecrosis, vascular or capsular invasion, Ki-67 labeling, and 


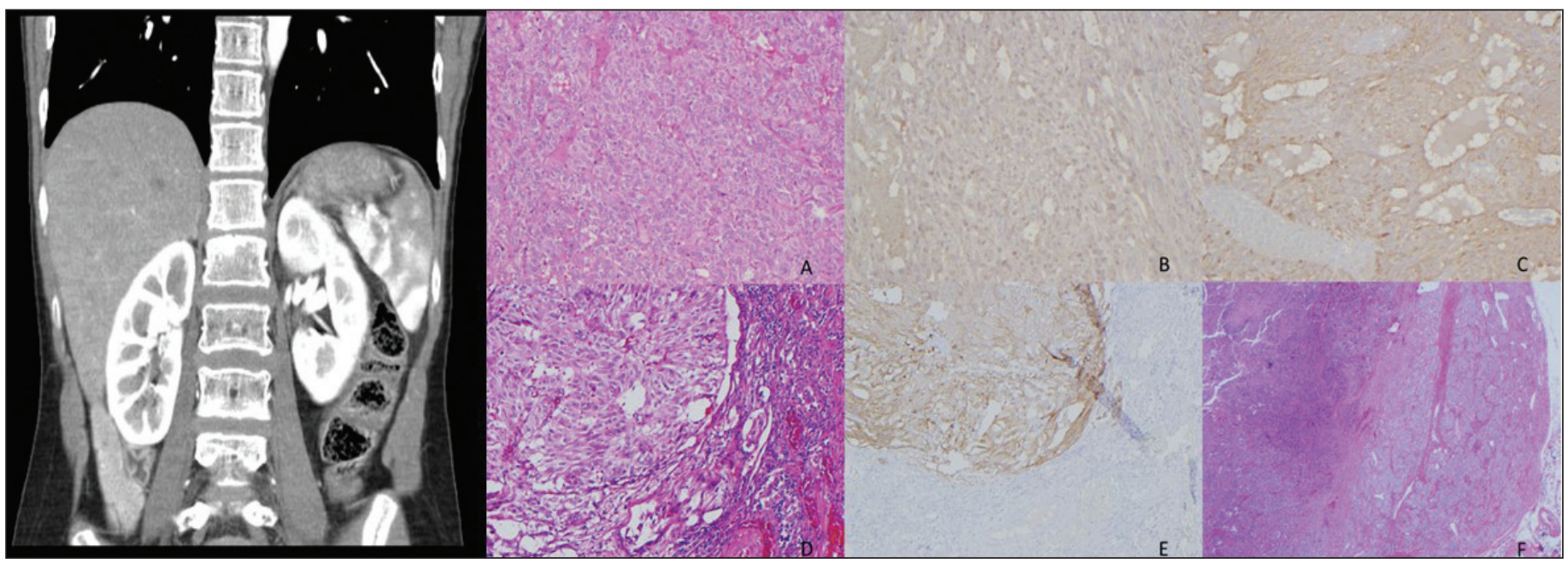

Fig 1. (Left) Computerized tomographic imaging showing a heterogeneous enhancing, solid cystic mass in the left adrenal gland measuring $4.3 \times 3.3 \times 3.0 \mathrm{~cm}$. The mass displaced the anterior margin of the upper pole of the left kidney without invasion into renal parenchyma. The left renal artery is displaced by the mass without significant compression. (Right) Pheochromocytoma, paraganglioma, and lymph node metastasis; (A) pheochromocytoma primary adrenal medullary tumour with characteristic Zellballen pattern and low-risk histopathology (H\&E); (B) synaptophysin positivity in main tumour; (C) chromogranin positivity in main tumour; (D) paraaortic paraganglioma (H\&E); (E) synaptophysin positivity in para-aortic paraganglioma; and (F) lymph node metastasis (H\&E).

catecholamine subtype. The tumour had a Zellballen pattern with low cellularity, with the absence of comedonecrosis, lymphovascular invasion, or high Ki-67. Although staining for the specific catecholamine subtype was not performed, this is considered well-differentiated.

One year following left adrenalectomy, the patient represented with symptoms of headaches, palpitations, and anxiety, with an increasing plasma normetanephrines, concerning for recurrence. Repeat positron-emission tomography (PET)-CT revealed interval growth of the right adrenal lesion and a new left para-aortic nodule at the left renal hilum. Familial PHEO/PGL syndrome was suspected and a staged, minimally invasive approach was planned with the first attempt to spare the right adrenal to prevent adrenal insufficiency, followed by removal of the left peri-aortic nodule with completion periaortic lymph node dissection.

After phenoxybenzamine blockade supplemented by metyrosine, the patient underwent robot-assisted laparoscopic partial right adrenalectomy. A $1.2 \mathrm{~cm}$ pheochromocytoma was identified with a focally positive resection margin consistent with enucleation and adrenal preservation surgery. Two months following the right partial adrenalectomy, a complete robot-assisted laparoscopic left-sided retroperitoneal node dissection was performed. Evaluation of 12 lymph nodes revealed a focus of metastatic pheochromocytoma measuring $1.2 \mathrm{~mm}$. The tumour metastasis, similar to the primary tumours, was well-differentiated and lacked adverse features. Four percent of well-differentiated/low-risk tumours metastasize. ${ }^{1}$ A lumbar puncture to evaluate for leptomeningeal spread was negative. Two years later, there is no evidence of residual disease biochemically or radiologically; the patient is asymptomatic and presumed in remission.
Due to young age, tumour recurrence, and metastatic disease, the possibility of a genetic mutation underlying tumorigenesis was considered. In addition to identifying an underlying genetic risk, molecular panels risk stratify PHEO/ PGL based on gene mutations, catecholamines, and signaling pathways. Those tumours driven by pseudohypoxia (mutations in SDH, FH, HIF, Ras, KIF1B, PHD2, or VHL) tend to produce norepinephrine or dopamine and behave aggressively. Pseudohypoxia associated pheochromocytomas have a $70 \%$ extra-adrenal involvement, multiple tumours in 30\%, high rate of metastases, and a majority are intermediate grade $(87 \%)$. Tumours driven by kinase signaling (mutations in Ret oncogene, NF1, TMEM127, or Max) produce epinephrine, are low-grade, and metastatic disease is infrequent. ${ }^{4}$

A next-generation sequencing panel evaluating genes associated with PHEO/PGL was performed (PGLNext panel, Ambry genetics). Genes on this panel include FH, MAX, MEN1, NF1, RET, SDHA, SDHAF2, SDHB, SDHC, SDHD, TMEM127, and VHL. Full gene sequencing and gross deletion/duplication analysis was performed and identified no pathological abnormalities. A comparative genomic hybridization study was also performed, and results from a SNP microarray (Affymetrix Cytoscan HD) did not identify any major copy number gains or losses and the karyotype was normal. Although the patient had bilateral and metastatic pheochromocytoma, no diseasecausing mutations were identified. It is likely that the history of BWS predisposed to tumour formation.

\section{Discussion}

BWS is an autosomal dominant disorder affecting 1 of 86000 individuals caused by altered expression of the 11 p15.5 locus 
due to differences in imprinting. The genes encoded at this locus include the genes IGF-R1, CDKN1C, and non-coding RNAs KCNQ1OT1 and H19. All of these loci regulate cell proliferation. BWS is associated with increased growth within the first few years of life, manifesting as hemihypertrophy or macroglossia. There is an increased rate of malignancy $(7.5-11 \%)^{5}$ and congenital malformations (abdominal wall defects and visceromegaly).

There are five reported cases associating BWS with development of pheochromocytoma, including three cases with bilateral adrenal disease. ${ }^{4,6-8}$ None of the previous studies performed prognostic gene sequencing panels or comparative genome hybridization to examine for underlying genomic mutations.

A multiomics approach of $>100$ pheochromocytomas revealed a low overall degree of genomic instability. ${ }^{4} 11 \mathrm{p}$ deletions were one of the most common copy number variants identified, including the 11 p15.5 locus ${ }^{4}$ that includes IGF-1R. IGF-1R is involved in cellular growth, contributing to carcinogenesis. Overexpression allows for anchorageindependent growth-promoting invasion and metastasis. There is cross-talk among other pathways, including that of the ER, EGFR, and human epidermal growth factor. ${ }^{9}$ This cross-talk leads to downstream mTOR signaling and RAFMEK activation. ${ }^{9}$ Therefore, BWS patients can be resistant to inhibitors of mTOR or the Ras-Raf-Mek-MAPK pathway due to upstream activation.

Interestingly, downstream signaling by IGFs includes multiple players in the pathogenesis of pheochromocytomas.
Therefore, although a genetic mutation in one of the known genes predisposing to pheochromocytomas and paragangliomas was not present, the upstream signaling elicited by IGF$1 \mathrm{R}$ can produce a similar cascade in patients with BWS. This cascade involves components of both the pseudohypoxia pathways (from mTOR activation and a metabolic shift mediated by the Warburg effect and dependence on glycolysis, associated with SDH and FH mutations) and kinase-signaling pathways. Due to upstream activation by insulin-like growth factors and other associated growth factors involved in the cross-talk (EGFR, HER-2, etc), IGF-1R tumours could have aggressive behaviour. Currently, IGF signaling is a target for cancer therapy ${ }^{10}$ and may act as a target of choice in BWS with metastatic disease. A proposed mechanism showing the cross-talk between IGF-1R with known pathways involved in the pathogenesis of pheochromocytomas and paragangliomas is shown in Fig. 2.

\section{Conclusion}

The genetic abnormality at chromosome locus 11 p15.5 appears to play a role in the tumorigenesis of pheochromocytoma in patients with BWS.

Competing interests: The authors report no competing personal or financial interests.

This paper has been peer-reviewed.

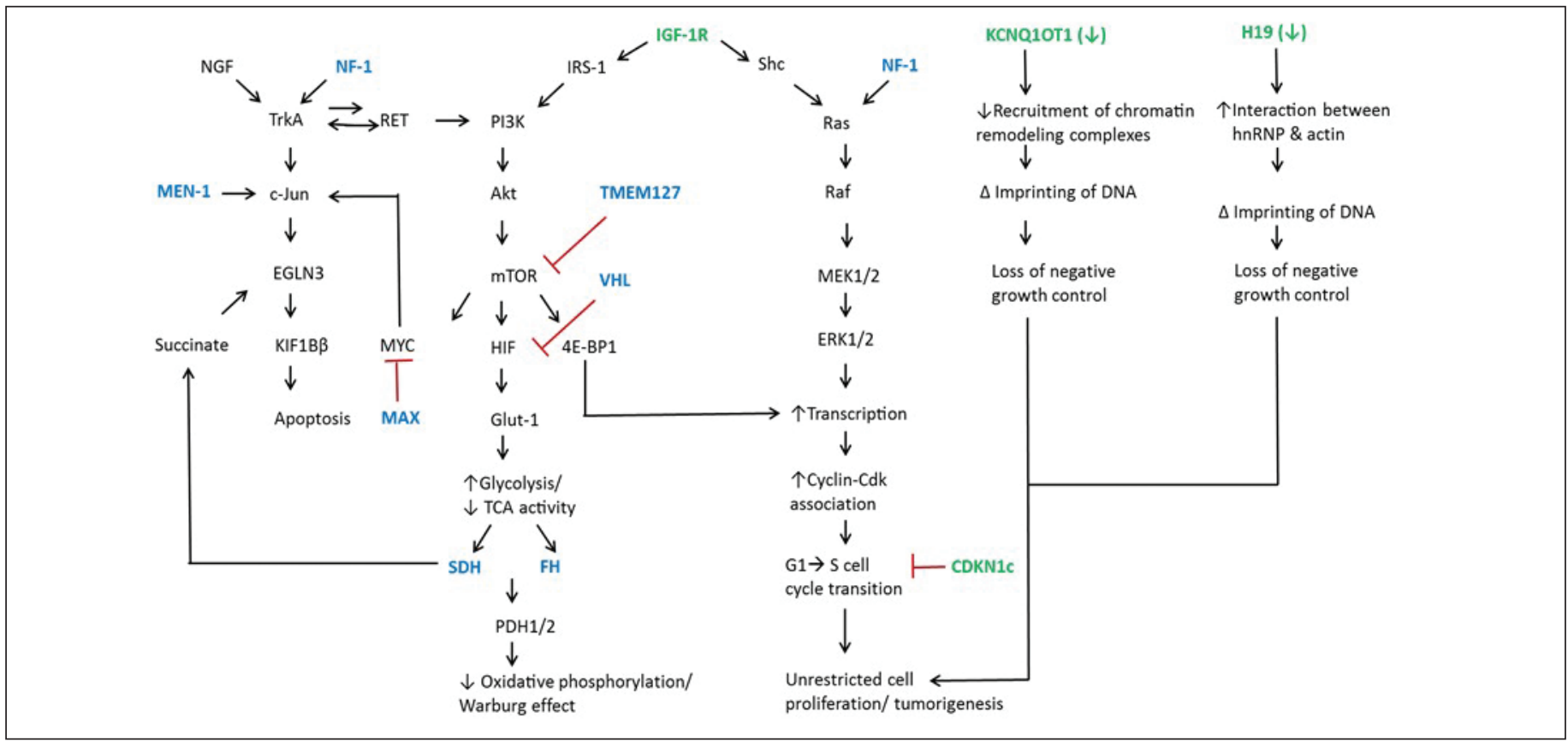

Fig. 2. Proposed signalling pathway showing that genetic loci deregulated through altered methylation in Beckwith-Wiedeman syndrome are upstream of pheochromocytoma-paraganglioma susceptibility genes. Green: protein or regulatory RNA encoded at chromosome 11p15.5; blue: proteins encoded by genes with known mutations in pheochromocytoma-paraganglioma; black arrows: positive regulation; red lines: negative regulation/silencing mechanisms. 


\section{References}

1. Tischler AS, deKriiger RR. Pathology of pheochromocytomas and paragangliomas. Endocr Relat Cancer 2015;22: T124-T133. https://doi.org/10.1530/ERC-15-0261

2. Kimura N, Takayanagi R, Takizawa N, et al. Pathological grading for predicting metastasis in phaeochromocytoma and paraganglioma. Endocr Relat Cancer 2014;21:405-14. https://doi.org/10.1530/ ERC-13-0494

3. Kimura N. A pathologist's view: Molecular profiles in diagnosing pheochromocytomas and paragangliomas. Int J Endo Oncol 2015;2:194-200. https://doi.org/10.2217/ije.15.16

4. Castro-Vega $\mathrm{L}$, Letouzé E, Burnichon N, et al. Multiomics analysis defines core genomic alterations in pheochromocytomas and paragangliomas. Nat Commun 2015;6.

5. hitps://doi.org/10.1038/ncomms7044

6. Wiedemann H. Tumours and hemihypertrophy associated with Wiedemann-Beckwith syndrome. Eur J Pediatr 1983;141:129. https://doi.org/10.1007/BF00496807
7. Baldisserotto M, Peletti AB, Ângelo de Araújo M, et al. Beckwith-Wiedemann syndrome and bilateral adrenal pheochromocytoma: Sonography and MRI findings. Pediatr Radiol 2005;35:1132-4. https://doi.org/10.1007/s00247-005-1518-3

8. Bémurat $L$, Gosse $P$, Ballanger $P$, et al. Successful laparoscopic operation of bilateral pheochromocytoma in a patient with Beckwith-Wiedemann syndrome. J Hum Hypertens 2002;16:281-4. https://doi.org/10.1038/si.jhh.1001378

9. Van den Akker EL, De Krijger RR, De Herder WW, et al. Congenital hemihypertrophy and pheochromocytoma, not a coincidental combination? Eur J Pediatr 2002;161:157-60. https://doi.org/10.1007/ s00431-001-0901-9

10. Chen HX, Sharon E. IGF-IR as an anti-cancer target-trials and tribulation. Chin J Cancer 2013;32:242-52. https://doi.org/10.5732/cic.012.10263

Correspondence: Dr. Tiffany Caza, Department of Pathology, Upstate Medical University, Syracuse, NY, United States; caza†@upstate.edu 